\title{
Serum levels of soluble CD25 as a marker for hepatocellular carcinoma
}

\author{
RONIEL CABRERA ${ }^{1}$, ASEM I. FITIAN ${ }^{2}$, MIGUEL ARARAT $^{1}$, YILING XU $^{1}$, TODD BRUSKO $^{3}$, \\ CLIVE WASSERFALL ${ }^{3}$, MARK A. ATKINSON $^{3}$, CHEN LIU $^{3}$ and DAVID R. NELSON ${ }^{1}$ \\ ${ }^{1}$ Section of Hepatobiliary Diseases, Department of Medicine; Departments of ${ }^{2}$ Molecular Genetics and Microbiology \\ and ${ }^{3}$ Pathology, Immunology and Laboratory Medicine, University of Florida, Gainesville, FL 32610-0214, USA
}

Received April 7, 2012; Accepted July 18, 2012

DOI: $10.3892 / \mathrm{ol} .2012 .826$

\begin{abstract}
In a previous study, we showed that the level of soluble CD25 (sCD25) was elevated in a small series of patients with hepatocellular carcinoma (HCC). In the present study, we determined the capacity of serum levels of sCD25 to detect the presence and the early stage of HCC using a larger cohort of HCC patients and evaluated the correlation between SCD25 level and tumor burden. Serum levels of sCD25 were quantified using ELISA in patients with HCC ( $n=145)$, controls with advanced fibrosis $(n=61)$ and healthy control subjects $(n=30)$. The levels of sCD25 in patients with HCC (median, 6,955 pg/ml) were significantly higher than those in cirrhosis-only patients $(4,310 \mathrm{pg} / \mathrm{ml} ; \mathrm{P}<0.0001)$. At a cut-off value of $2,180 \mathrm{pg} / \mathrm{ml}$, sCD25 had a sensitivity of $92.3 \%$ and a specificity of $37.7 \%$ in detecting HCC presence [area under the curve (AUC) of 0.685 ; $\mathrm{P}<0.0001]$. By comparison, $\alpha$-fetoprotein (AFP) had a sensitivity of $53.8 \%$ and a specificity of $86.8 \%$ at a cut-off value of $32.8 \mathrm{ng} / \mathrm{ml}(\mathrm{AUC}=0.755 ; \mathrm{P}<0.0001)$ for $\mathrm{HCC}$ presence detection. For early HCC, the sensitivity of sCD25 was $89.6 \%$ and its specificity was $39.3 \%$ (AUC $=0.630 ; \mathrm{P}<0.0001)$ at a cut-off value of $2,859 \mathrm{pg} / \mathrm{ml}$, while AFP had a sensitivity of $41.7 \%$ and a specificity of $82.6 \%$ at a cut-off value of $20.6 \mathrm{ng} / \mathrm{ml}$ (AUC=0.630; $\mathrm{P}=0.0257$ ). We also found a significant positive correlation between serum levels of sCD25 and tumor stage. In the present study study, sCD25 was more effective than AFP at detecting the presence and early stages of HCC. This immune factor may hold promise as a novel predictive marker of HCC presence and may be useful in distinguishing early HCC from advanced cirrhosis, currently areas of global unmet need.
\end{abstract}

Correspondence to: Dr Roniel Cabrera, Section of Hepatobiliary Diseases, Department of Medicine, University of Florida, 1600 SW Archer Road, PO Box 100214, Gainesville, FL 32610-0214, USA

E-mail: roniel.cabrera@medicine.ufl.edu

Key words: biomarker, soluble CD25, hepatocellular carcinoma

\section{Introduction}

Hepatocellular carcinoma (HCC) is among cancers with the poorest outlook, with fewer than $12 \%$ of all patients surviving at five years (1). Primary liver cancer most often emerges as a complication of chronic liver disease, specifically cirrhosis. In western countries, the most common cause of cirrhosis is presently chronic hepatitis $\mathrm{C}$ virus (HCV) infection and non-alcoholic fatty liver disease (NAFLD) is emerging as a primary risk factor due to the epidemic of obesity (2). In a population with cirrhosis, the most common clinical sequela is HCC (3). The early detection of HCC markedly improves outcome (4). While the risk factors for patients at the highest risk for developing HCC are well characterized, the early detection of liver cancer remains a challenge. The current screening tools of ultrasonography and measurement of $\alpha$-fetoprotein (AFP) in the blood have limitations $(5,6)$. In clinical practice, AFP is not elevated in a significant number of patients with HCC (7). While the serological AFP blood test is non-invasive, inexpensive and reproducible, screening programs that utilize the test suffer from limitations in this marker's sensitivity and specificity. The poor sensitivity of AFP explains its absence from the AASLD practice guidelines as a test recommended for screening of HCC (8). This substandard sensitivity underlines the need for a biomarker that is able to detect HCC at an early stage.

The immune system employs various defense mechanisms to inhibit cancer proliferation. However, a hallmark of cancer is the ability to exploit these defenses and ultimately eclipse tumor immunity (9). We have identified soluble CD25 (sCD25) as an immune factor that is part of the immune-suppressive network of HCC with potential promise as a biomarker for HCC. sCD25 is produced after proteolytic release from the membrane-bound $\alpha$-subunit (CD25) of the interleukin (IL)-2 receptor. When $\mathrm{CD} 25$ is present on the T-cell membrane, together with the $\beta$ and $\gamma$ chains it forms the high-affinity IL-2 receptor that allows optimal IL-2 signaling for T-cell activation and proliferation (10). We have previously shown that the level of sCD25 in the serum of patients with HCC is directly correlated with the degree of tumor burden (11). In addition to the correlation with HCC burden, sCD25 also has novel functional properties with an ability to inhibit, in a dose-related manner, antitumor T-cell responses. The release 
of sCD25 with its immune-inhibitory properties is another level of immune regulation involved in $\mathrm{HCC}$ development and progression (12-14).

In the present study, we evaluated the correlation between the serum level of sCD25 and liver pathology using a welldefined cohort of patients with HCC and patients with advanced liver fibrosis. We hypothesized that $\mathrm{sCD} 25$ has the potential to be an effective biomarker for the presence and early detection of HCC. We determined the level of sCD25 in healthy subjects (NCs), disease controls (DCs) with advanced fibrosis and patients with HCC. We then determined the sensitivity and specificity of sCD25 in order to distinguish patients with HCC from controls with advanced fibrosis and cirrhosis. We also evaluated the efficacy of SCD25 in detecting early HCC. We concluded our study by revisiting the connection between sCD25 level and tumor burden observed in our previous study using this larger cohort of patients with HCC.

\section{Materials and methods}

Study population. The study protocol was approved by the University of Florida Institutional Review Board and we obtained written informed consent from all participants in the study. The study included 143 patients with HCC in the setting of cirrhosis, 61 liver DCs and 30 NCs. Cirrhosis in the HCC patients developed from various primary etiologies (Table IB). The DC etiologies included patients with chronic HCV or $\mathrm{HBV}$ infection, chronic HCV- or HBV-related cirrhosis, $\mathrm{HCV}$ and HBV co-infection, alcohol abuse, NAFLD and cryptogenic cirrhosis (Table IA). All DCs were evaluated for stage of fibrosis with a liver biopsy and serum collection was performed on the same day as the biopsy. Of the 61 DCs, 54 had cirrhosis and these patients had a fibrosis stage $\geq 3$ out of 6 (Table IA) (15). All DCs with cirrhosis and patients with HCC enrolled in this study had well-compensated cirrhosis compatible with Child-Pugh A classification. No patients had a performance status $>1$ and the majority of HCC patients had a performance status score of 0 . Blood samples from genderand age-matched NCs ( $n=30 ; 15$ males, 15 females) were obtained from the local blood bank (Life South, Gainesville, FL, USA). HCC was diagnosed according to the non-invasive radiological criteria per the AASLD guidelines (7). The staging of HCC was performed using the Barcelona Clinic Liver Cancer (BCLC) staging system. Patients diagnosed with stage A HCC had a single lesion $<5 \mathrm{~cm}$ or $2-3$ lesions $<3 \mathrm{~cm}$ in size. Multinodular lesions $>5 \mathrm{~cm}$ were characteristic tumor features in patients with stage B HCC. Macroscopic vascular invasion or metastatic disease was established in patients with Stage C HCC (16).

Within the group of DCs, 44 patients had HCV-related cirrhosis confirmed by histopathology. The patients with confirmed HCV-related cirrhosis were enrolled into our surveillance program, received serial cross-sectional imaging every six months and had no liver masses on enrollment and 12 months after enrollment. The following clinical data were obtained for each HCC patient: age, gender, ethnicity, etiology of HCC, BCLC stage, AFP level and Model for End-Stage Liver Disease (MELD) score. For the DCs, we obtained age, gender, ethnicity, etiology of liver disease, MELD score and AFP data for 46 of the 61 DC patients. AFP was measured in 46 of the 61 DC patients. Laboratory hepatic function data needed for MELD score calculation was not obtained for 10 of the 61 DCs at the time of their enrollment

Serum preparation and SIL-2R ELISA for sCD25 quantification. Whole blood samples were collected on the clinic date when patients were diagnosed with HCC and processed for serum isolation. Then, using sIL-2R ELISA (Bender MedSystems, Vienna, Austria), we processed fresh samples for sCD25 in duplicate using our previous approach (11). Briefly, microtiter plates coated with anti-human sIL-2R antibody were inculcated with serum containing SCD25 and subjected to horseradish peroxide, a substrate solution that upon addition induced a color change. The intensity of the colored product was directly proportional to the level of sCD25 present in each well. Plates were read at $450 \mathrm{~nm}$ using the SpectraMax 190 reader (Molecular Devices, Sunnyvale, CA, USA). Measurements of sCD25 above the upper limit of the calibration range $(20,000 \mathrm{pg} / \mathrm{ml})$ were diluted by half using buffer from the manufacturer.

Statistical analysis. Data for sCD25 and AFP levels are expressed as box plots with medians \pm SD. Receiver operator characteristic (ROC) curves with respective points of maximal accuracy for sensitivity and specificity were generated to determine biomarker performance. The multiple regression test was used to evaluate the correlation between clinical parameters and sCD25 level. We used the Mann-Whitney U test to assess the significance of group differences in the level of sCD25. Spearman's rank correlation coefficient was used to examine the correlation between HCC stage and the level of sCD25. $\mathrm{P}<0.05$ was considered to indicate a statistically significant result. Statistical data were analyzed using MedCalc version 11.5.1.0 (MedCalc Software, Mariakerke, Belgium).

\section{Results}

Clinical characteristics of HCC patients and DCs. In our study, the majority of HCC patients were male $(79 \%)$ and Caucasian (82\%). African-Americans, Hispanics and Asians were less common (Table I). A near-equal distribution of patients was present across all stages of $\mathrm{HCC}$, with 48 patients having stage A disease (early HCC), 45 patients being in the stage B subset (intermediate $\mathrm{HCC}$ ) and 50 patients having stage $\mathrm{C}$ cancer (advanced HCC). The average age of the HCC patients was 63.6 years. Of the HCC patients, $60 \%$ had chronic HCV infection, making $\mathrm{HCV}$ the predominant etiology of their liver disease. Other primary causes of HCC included alcohol-related cirrhosis $(n=13)$, chronic HBV infection $(n=7)$ and NAFLD/non-alcoholic steatohepatitis (NASH; $n=13$ ). The majority of HCC patients (97\%) had a MELD score $<15$. Most HCC patients $(67.13 \%)$ had an AFP level $<400 \mathrm{ng} / \mathrm{ml}$. For the DCs, the main etiology of their liver disease was chronic HCV infection and nearly all patients had an AFP level <400 ng/ml (Table IB).

sCD25 levels in HCC patients. The serum levels of sCD25 were detected at a significantly higher level in HCC patients than NCs and DCs (Fig. 1A). The median value of sCD25 in the $\mathrm{HCC}$ patients $(6,955 \mathrm{pg} / \mathrm{ml})$ was significantly higher than that of the DCs and NCs $(\mathrm{P}<0.0001)$. The median level of 
Table I. Baseline clinical characteristics of HCC $(n=143)$ and disease control patients.

A, Disease controls $(n=61)$

\begin{tabular}{|c|c|}
\hline Category & $\mathrm{n}(\%)$, or mean (range) \\
\hline Age (years) & $55.9(45-77)$ \\
\hline \multicolumn{2}{|l|}{ Gender } \\
\hline Male & $42(68.90)$ \\
\hline Female & $19(31.15)$ \\
\hline \multicolumn{2}{|l|}{ Ethnicity } \\
\hline Caucasian & $53(86.89)$ \\
\hline African-American & $5(8.20)$ \\
\hline Hispanic & $2(3.28)$ \\
\hline Asian & $1(1.64)$ \\
\hline \multicolumn{2}{|l|}{ Etiology } \\
\hline Cryptogenic cirrhosis & $2(3.28)$ \\
\hline EtOH cirrhosis & $3(4.92)$ \\
\hline $\mathrm{HBV}$ & $1(1.64)$ \\
\hline HBV cirrhosis & $1(1.64)$ \\
\hline $\mathrm{HBV}+\mathrm{HCV}$ cirrhosis & $1(1.64)$ \\
\hline $\mathrm{HCV}$ & $6(9.84)$ \\
\hline $\mathrm{HCV}$ cirrhosis & $44(72.13)$ \\
\hline NAFL cirrhosis & $3(4.92)$ \\
\hline \multicolumn{2}{|l|}{ AFP } \\
\hline$\leq 400 \mathrm{ng} / \mathrm{ml}$ & $45(97.83)$ \\
\hline$>400 \mathrm{ng} / \mathrm{ml}$ & $1(2.17)$ \\
\hline \multicolumn{2}{|l|}{ MELD score } \\
\hline$<10$ & $40(78.43)$ \\
\hline$\geq 10$ & $11(21.57)$ \\
\hline
\end{tabular}

B, HCC patients $(n=143)$

\begin{tabular}{lc}
\hline Category & $\mathrm{n}(\%)$, or mean (ran \\
\hline Age (years) & $63.6(30-92)$ \\
Gender & \\
Male & $113(79.02)$ \\
Female & $30(20.98)$ \\
Ethnicity & \\
Caucasian & $117(81.82)$ \\
African-American & $15(10.49)$ \\
Hispanic & $8(5.59)$ \\
Asian & $3(2.10)$ \\
Etiology & \\
Adenoma & $4(2.80)$ \\
Cryptogenic cirrhosis & $19(13.29)$ \\
EtOH cirrhosis & $13(9.09)$ \\
HBV cirrhosis & $7(4.90)$ \\
HCV cirrhosis & $86(60.14)$ \\
NAFL cirrhosis & $13(9.09)$ \\
PBC cirrhosis & $1(0.70)$
\end{tabular}

Table IB. Continued.

\begin{tabular}{lr}
\hline B, HCC patients $(\mathrm{n}=143)$ & \\
\hline Category & $\mathrm{n}(\%)$, or mean (range) \\
\hline HCC stage $^{\mathrm{a}}$ & \\
A & $48(33.57)$ \\
B & $45(31.47)$ \\
C & $50(34.97)$ \\
AFP & \\
$<400 \mathrm{ng} / \mathrm{ml}$ & $96(67.13)$ \\
$>400 \mathrm{ng} / \mathrm{ml}$ & $47(32.87)$ \\
MELD score & \\
$<10$ & $106(74.13)$ \\
$\geq 10$ & $37(25.87)$ \\
\hline
\end{tabular}

a Stage based on the Barcelona Clinic Liver Cancer HCC staging system. HCC, hepatocellular carcinoma; HBV, hepatitis B virus; $\mathrm{HCV}$, hepatitis C virus; NAFL, non-alcoholic fatty liver; PBC, primary biliary cirrhosis; AFP, $\alpha$-fetoprotein; MELD, Model for EndStage Liver Disease.

$\mathrm{sCD} 25$ in the DC group $(4,310 \mathrm{pg} / \mathrm{ml})$ was higher than that in the NCs $(2,098 \mathrm{pg} / \mathrm{ml})$, but the difference was not significant $(\mathrm{P}=0.0676)$. The levels of $\mathrm{sCD} 25$ of HCC patients were not correlated with age, gender or MELD score.

AFP levels in HCC patients. AFP levels were obtained for DC and HCC patients but not the NCs. The difference between the median AFP level in HCC patients $(49.4 \mathrm{ng} / \mathrm{ml})$ and that in DCs $(5.3 \mathrm{ng} / \mathrm{ml})$ was significant $(\mathrm{P}<0.0001$; Fig. 1B). For the $\mathrm{DC}$ and HCC patients, there was no correlation between the AFP levels and age, gender or MELD score.

Utility of sCD25 in predicting HCC presence. After showing that $\mathrm{sCD} 25$ was able to differentiate $\mathrm{HCC}$ from controls with cirrhosis $(\mathrm{P}<0.0001)$, we then determined its capacity to detect the presence of HCC. This analysis showed that at a cut-off value of $2,180 \mathrm{pg} / \mathrm{ml}, \mathrm{sCD} 25$ had a sensitivity of $92.3 \%$ and a specificity of $37.7 \%$ for detecting HCC (Fig. 2A). By comparison, AFP had a sensitivity of $53.8 \%$ and a specificity of $86.8 \%$ at a cut-off value of $32.3 \mathrm{ng} / \mathrm{ml}$. The area under the curve (AUC) values for SCD25 and AFP were 0.685 and 0.755 , respectively. At $20 \mathrm{ng} / \mathrm{ml}$, the recommended clinical cut-off value for AFP used in clinical practice, the sensitivity of AFP was $60.1 \%$ and the specificity was $81.8 \%(\mathrm{AUC}=0.733)$.

sCD25 as a marker for early stage HCC. We evaluated the performance of sCD25 in detecting early HCC by comparing the level of sCD25 in patients with BCLC stage A HCC with the $\mathrm{sCD} 25$ responses of DC patients (Fig. 3A). In this ROC analysis, an optimal cut-off value of $2,859 \mathrm{pg} / \mathrm{ml}$ for sCD25 had a sensitivity of $89.6 \%$ and a specificity of $39.3 \%$ with an AUC of $0.630(\mathrm{P}<0.0001)$. By comparison, at a cut-off value of $20 \mathrm{ng} / \mathrm{ml}$, AFP had a sensitivity of $41.7 \%$ and a specificity of $82.6 \%$ (AUC=0.630, $\mathrm{P}=0.0257$; Fig. 3B). 

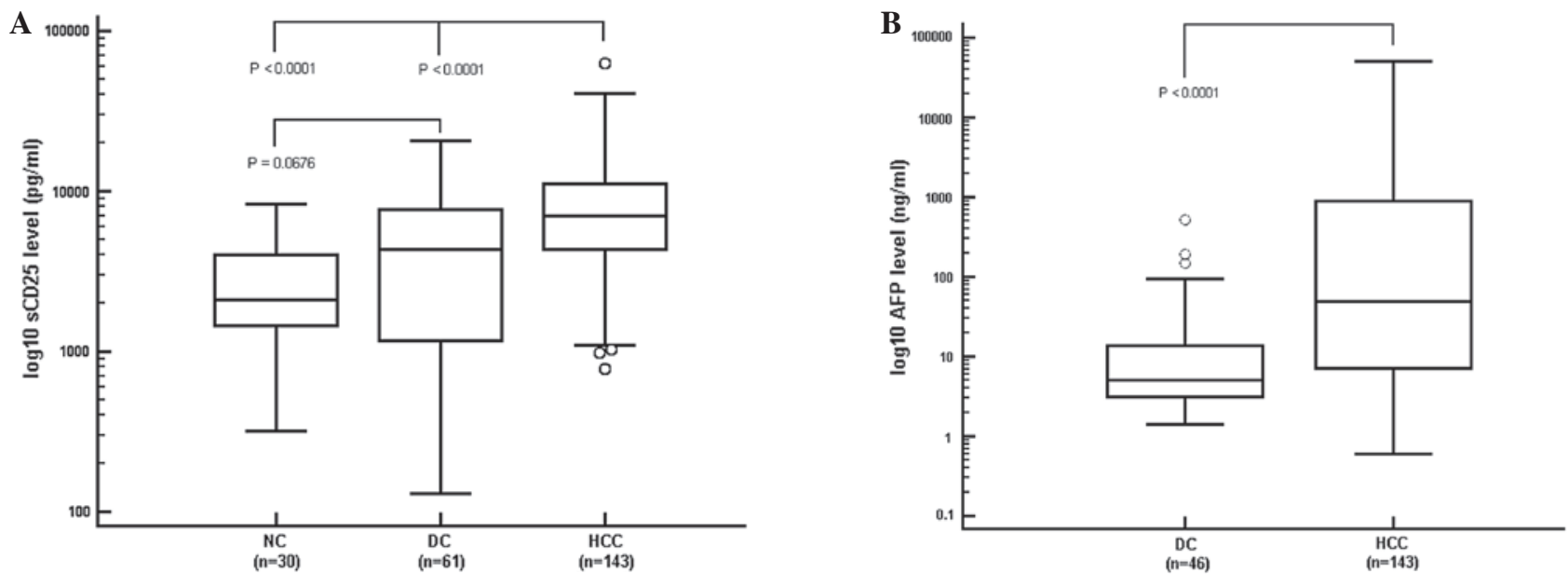

Figure 1. Serum levels of sCD25 and AFP. Values expressed as median \pm standard deviation. (A) Serum sCD25 level in HCC patients (6,955 pg/ml) was significantly higher than levels manifested in NCs $(2,098 \mathrm{pg} / \mathrm{ml})$ and DCs $(4,310 \mathrm{pg} / \mathrm{ml} ; \mathrm{P}<0.0001)$. (B) Serum AFP rose as liver disease worsened. DC patients had a median AFP concentration of $5.3 \mathrm{ng} / \mathrm{ml}$ and the median AFP level in HCC patients was $49.4 \mathrm{ng} / \mathrm{ml}$. sCD25, soluble CD25; AFP, $\alpha$-fetoprotein; HCC, hepatocellular carcinoma; NC, healthy control; DC, disease control.

A

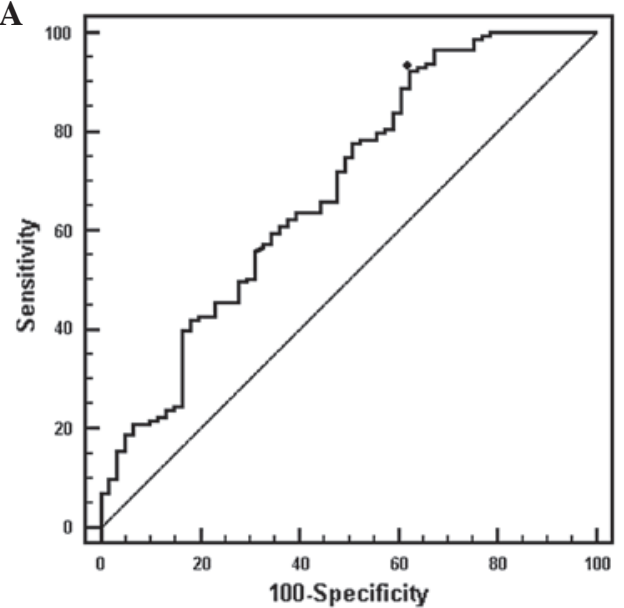

B

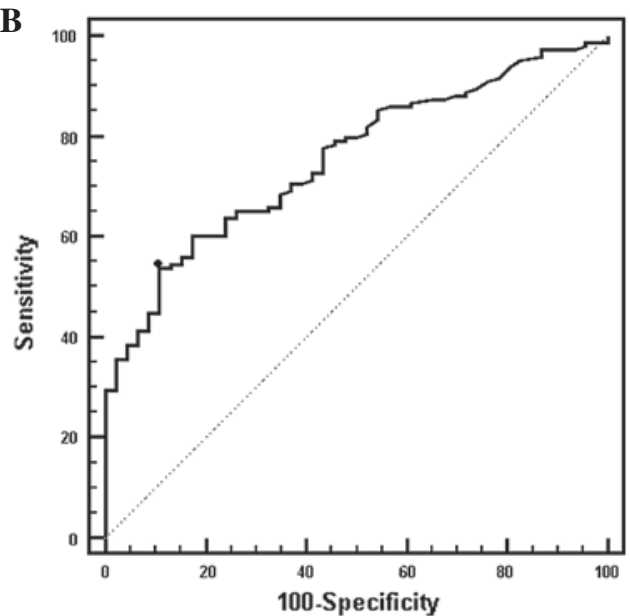

Figure 2. ROC curves: HCC presence. ROC curves demonstrate the potential of sCD25 as a predictor of HCC presence. (A) At a cut-off value of 2,180 pg/ml the sensitivity of sCD25 for $\mathrm{HCC}$ presence was $92.3 \%$ and the specificity was $37.7 \%$ (AUC=0.685). (B) Sensitivity of AFP was lower than the sensitivity for sCD25. At a cut-off value of $39.7 \mathrm{ng} / \mathrm{ml}$, the sensitivity of AFP was $53.1 \%$ while its specificity was $89.1 \%$ (AUC $=0.753$ ). ROC, receiver operator characteristic; sCD25, soluble CD25; HCC, hepatocellular carcinoma; AUC, area under the ROC curve; AFP, $\alpha$-fetoprotein.
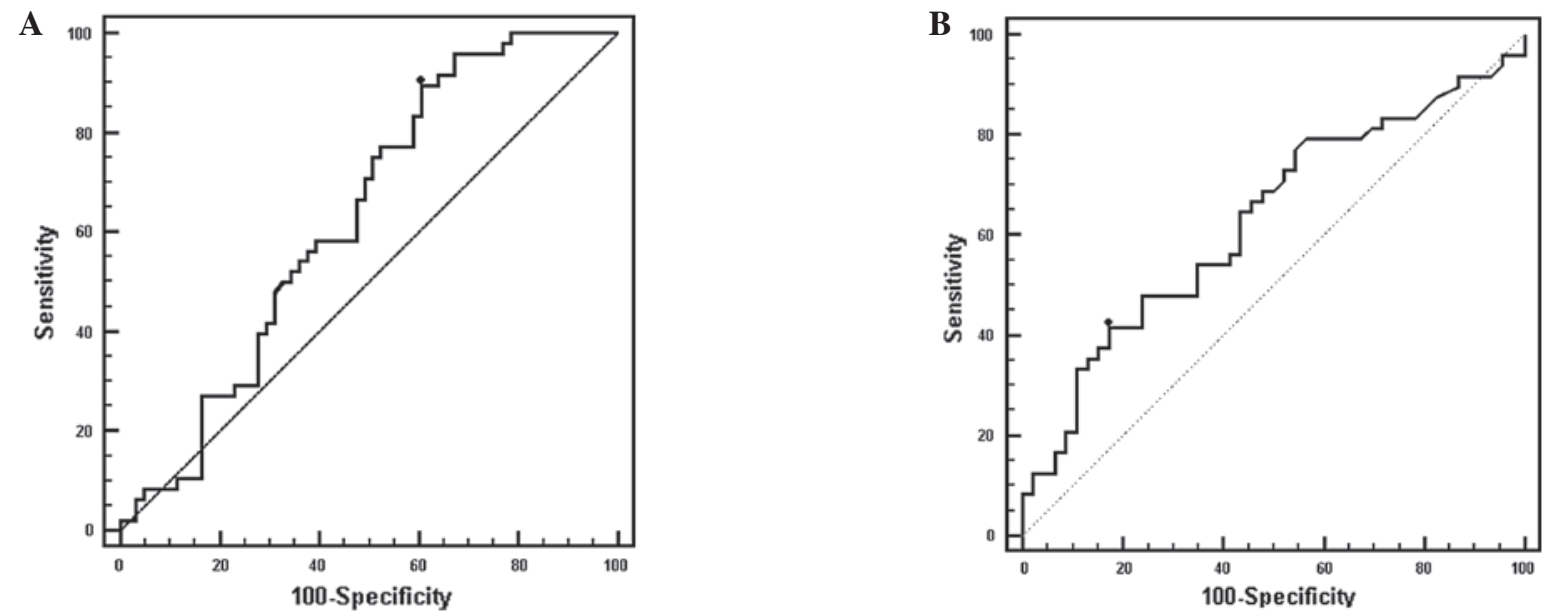

Figure 3. ROC curves: Early HCC. (A) ROC curve showing sensitivity and specificity of sCD25 in the detection of early stage HCC. The cut-off value of $2,859 \mathrm{pg} / \mathrm{ml}$ for sCD25 had a sensitivity of $89.6 \%$ and a specificity of $39.3 \%$ (AUC=0.630). (B) At a cut-off value of $20.6 \mathrm{ng} / \mathrm{ml} \mathrm{AFP}$ possessed a sensitivity of $41.7 \%$ and specificity of $82.6 \%$ ( $\mathrm{AUC}=0.630$ ). sCD25 demonstrated a stronger capacity to predict early cancer $(\mathrm{P}<0.0001)$ than $\mathrm{AFP}(\mathrm{P}=0.0257)$. ROC, receiver operator characteristic; HCC, hepatocellular carcinoma; sCD25 soluble CD25; AUC, area under the ROC curve; AFP, $\alpha$-fetoprotein. 

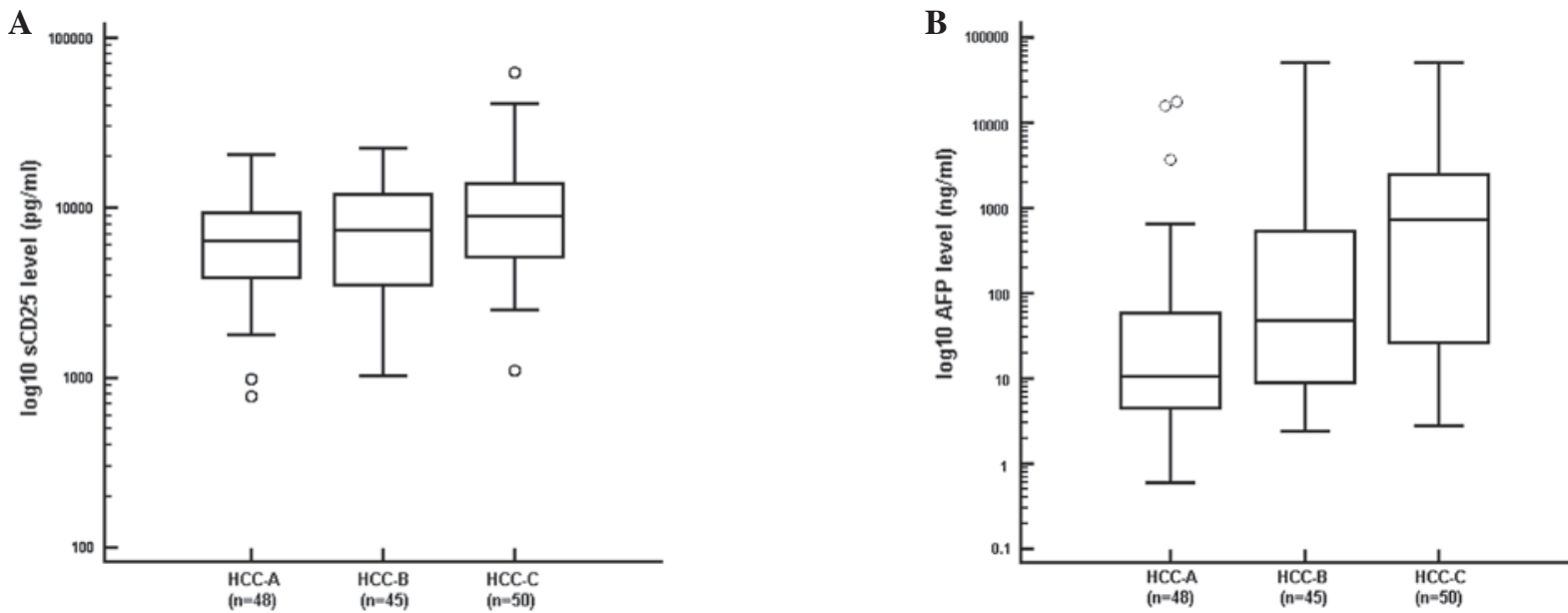

Figure 4. Tumor burden and sCD25 level. (A) Tumor burden was directly correlated with serum sCD25 level. (B) Tumor burden and serum AFP level increased in tandem. sCD25, soluble CD25; AFP, $\alpha$-fetoprotein; HCC, hepatocellular carcinoma. Stage based on the Barcelona Clinic Liver Cancer HCC staging system.

Table II. Correlation between sCD25 and stage A HCC tumor size.

\begin{tabular}{llcc}
\hline Tumor size $(\mathrm{cm})$ & Parameter & sCD25 & $\mathrm{AFP}^{\mathrm{a}}$ \\
\hline$<2$ & Sensitivity & 91.7 & 33.3 \\
& Specificity & 41.0 & 76.1 \\
$<5$ & Sensitivity & 92.1 & 47.4 \\
& Specificity & 39.3 & 82.6 \\
\hline
\end{tabular}

${ }^{a}$ Cut-off value, $2,859 \mathrm{pg} / \mathrm{ml}$; ${ }^{\mathrm{b}}$ cut-off value, $20.0 \mathrm{ng} / \mathrm{ml}$. sCD25, soluble CD25; HCC, hepatocellular carcinoma; AFP, $\alpha$-fetoprotein; stage based on the Barcelona Clinic Liver Cancer HCC staging system.

Correlation between tumor burden and $s$ CD25 level. We evaluated the correlation between levels of sCD25 and tumor burden. We also examined the correlation between AFP and tumor burden. We observed a progressive elevation in the level of $\mathrm{sCD} 25$ with increasing HCC stage $(\mathrm{R}=0.213$, $\mathrm{P}<0.0160$ ). The patients with early stage HCC (stage A) had the lowest median level of sCD25 $(6,339 \mathrm{pg} / \mathrm{ml})$, while patients with multi-nodular HCC (stage B) had an intermediate median level of $\operatorname{sCD} 25(7,365 \mathrm{pg} / \mathrm{ml})$. The patients with advanced HCC (stage $\mathrm{C}$ ) had the highest median level of sCD25 (8,889 pg/ml; Fig. 4A). We also found a strong positive correlation between AFP level and stage of HCC (Fig. 4B; $\mathrm{R}=0.513, \mathrm{P}<0.0001$ ).

Using the cut-off value of $2,859 \mathrm{pg} / \mathrm{ml}$ for $\mathrm{sCD} 25$ and $20 \mathrm{ng} / \mathrm{ml}$ for AFP, we evaluated the correlation between sCD25 and size of HCC in patients with early HCC. In this analysis, we divided the subset of HCC patients with early HCC into patients with a single small tumor $<2 \mathrm{~cm}$ and patients with single tumors $<5 \mathrm{~cm}$ in size. In this analysis, sCD25 retained a high sensitivity in those patients with the smallest lesions $(<2 \mathrm{~cm})$ and in patients with lesions $<5 \mathrm{~cm}$ (Table II). We also found that the sensitivity for AFP was low and increased with size of HCC.

\section{Discussion}

Given that the majority of HCC patients present with advanced disease, there is a pressing need for an effective biomarker that detects the presence and early stages of HCC at a better capacity than AFP. In the present study, we found that sCD25 effectively distinguished HCC patients from healthy and DC subjects. The levels of sCD25 in HCC patients were significantly higher than those in NCs and DCs with advanced liver fibrosis. This analysis found that $\mathrm{SCD} 25$ possesses a sensitivity of $92 \%$ at a cut-off value of 2,180 pg/ml for the presence of $\mathrm{HCC}$ and warrants additional investigation as a potential screening test. Furthermore, sCD25 also retained this high sensitivity (90\% at a cut-off value of 2,899 pg/ml) for detecting $\mathrm{HCC}$ in a subset of patients with early stage $\mathrm{HCC}$, highlighting its potential use as a screening tool in those at high-risk for HCC. When comparing sCD25 with AFP, we found that sCD25 has a higher sensitivity than AFP in detecting the presence of HCC, particularly in patients with early HCC. We also observed a positive correlation between the level of sCD25 and the degree of tumor burden of HCC, with levels of sCD25 progressively increasing from early (stage A) to advanced stage (stage C) HCC. This correlation was consistent with our previous study (11), which revealed a positive correlation between serum levels of sCD25 and tumor burden. These findings suggest that the measurement of serum levels of the immune marker sCD25 may improve earlier detection of HCC and could potentially be a useful novel prognostic marker.

A number of serum markers have been evaluated to detect HCC, including AFP, lectin-bound AFP (AFP-L3\%) and des- $\gamma$ carboxy-prothrombin (DCP). The most commonly used serological assay to detect HCC is the blood test for AFP. However, AFP at the clinically recommended cut-off value of $20 \mathrm{ng} / \mathrm{ml}$ suffers from poor sensitivity (17). Studies have suggested DCP and AFP-L3\% as potential biomarkers for HCC, particularly when used in a complementary fashion (18-20). However, a multicenter phase II biomarker study, using a total of 836 patients with $50 \%$ of the patients being controls with cirrhosis and $50 \%$ having HCC, showed the sensitivity of $60 \%$ for AFP to be better than those of DCP and AFP-L3\% (21). For those patients with early stage $\mathrm{HCC}$, the sensitivity of $65 \%$ for AFP 
was more sensitive than DCP and AFP-L3\%. Studies that combined the three markers AFP-L3\%, DCP and AFP have failed to show a substantial improvement in sensitivity, even when using a cohort of HCC patients with large, unresectable tumors (22). When patients with early HCC were analyzed from a nested case-control study of $39 \mathrm{HCC}$ cases developing during the randomized Hepatitis C Antiviral Long-term Treatment (HALT-C) trial, the sensitivity dropped to $47 \%$ for DCP and $61 \%$ for AFP (23). Numerous studies have shown that the sensitivity of these markers drops as a function of decreasing tumor size, highlighting an insufficient sensitivity for these markers at detecting the onset of cancer at its earliest stage (24-26). The need to be able to detect HCC at its earliest stage was further demonstrated in the HALT-C trial, which analyzed the serum levels of AFP and DCP for 12 months prior to the diagnosis of HCC. DCP and AFP had good specificities (94 and 75\%, respectively), but possessed markedly low sensitivities, of 47 and $43 \%$, respectively. In the present study, sCD25 was more sensitive than AFP in distinguishing patients with early HCC from cirrhosis control patients. Moreover, our study showed that AFP level was not correlated with early stage HCC lesions smaller than $3 \mathrm{~cm}(\mathrm{P}=0.1148)$. This finding is consistent with the conclusions of other studies (27) demonstrating a lack of sensitivity of the AFP serological test when used in the screening for early tumors.

While AFP had poor sensitivity in our study, it did show a high specificity for both HCC presence and early HCC in comparison to the low specificity of $\mathrm{sCD} 25$ in these analyses. The inadequate specificity of $\mathrm{sCD} 25$ is a limitation that requires further evaluation through the recruitment of a larger HCC cohort, since an ideal biomarker should possess high sensitivity and specificity. The addition of complementary markers to sCD25 should also be considered, since this approach to screening may improve the capacity of a test to detect cancer in its early stage.

AFP continues to be widely used but concern over its poor performance as a marker has led to its exclusion as a recommended test for screening patients at high risk for $\mathrm{HCC}$ in the current practice guidelines from the AASLD $(7,28,29)$. Currently, the main screening strategy recommended is serial liver ultrasonography. However, this screening modality is not being effectively used since the majority of patients at the highest risk for HCC development are not undergoing surveillance (30). Ultrasonography also poses significant challenges related to availability and operator experience in interpreting images from cirrhotic livers and obese patients (31). The identification of a novel biomarker for HCC that detects early cancer and is capable of overcoming these limitations may improve surveillance efforts and clinical outcomes.

While this study was not designed to evaluate the influence of the etiology of underlying liver disease or other clinical factors on SCD25, we did not identify a correlation between clinical parameters and levels of sCD25 level. Most importantly, our study controlled for underlying liver function by enrolling only HCC patients with Child-Pugh A cirrhosis. Biases were further eliminated through the blind implementation of bioassay procedures. Our study demonstrated a dose-response correlation between sCD25 level and tumor burden, suggesting its potential use as predictor of prognosis at baseline. Future studies analyzing sCD25 responses in samples obtained during a surveillance program may provide further insight on the utility of sCD25 in surveillance for early HCC detection.

We currently lack a reliable serum marker for the early detection of HCC. In accordance with the phase-specific biomarker standardization model delineated by Pepe et al, we highlight the progress of our initial study using the immune marker of sCD25 (32). Previously, we assessed the performance of sCD25 in a small group $(n=60)$ of HCC patients. In the present study, we expanded our analysis to a larger cohort of HCC patients $(n=143)$ and again observed the previously shown marked elevation of sCD25 in HCC patients in comparison to the levels manifested in healthy controls and controls with cirrhosis (11). Our findings show that sCD25 distinguished HCC from appropriate controls and that this marker identified the presence of HCC more effectively than AFP, particularly in patients with early tumors. The high sensitivity of sCD25 suggests it holds promise as a marker for early HCC which is an area of unmet need. To further characterize the utility of sCD25 in detecting early stages of HCC tumor development, larger longitudinal and validation studies are planned.

\section{Acknowledgements}

This study was supported by the NIH KL2 University of Florida Clinical Translational Science Scholar Award, NIH/NCRR award UL1RR029890 and NIH/NCI award K24CA139570. The authors thank the participants of this study for their dedication and commitment.

\section{References}

1. El-Serag HB: Hepatocellular carcinoma. N Engl J Med 365: 1118-1127, 2011.

2. Caldwell SH, Crespo DM, Kang HS and Al-Osaimi AM: Obesity and hepatocellular carcinoma. Gastroenterology 127 (Suppl 1): S97-S103, 2004.

3. Fattovich G, Stroffolini T, Zagni I and Donato F: Hepatocellular carcinoma in cirrhosis: incidence and risk factors. Gastroenterology 127 (Suppl 1): S35-S50, 2004.

4. Wong LL, Limm WM, Severino R and Wong LM: Improved survival with screening for hepatocellular carcinoma. Liver Transpl 6: 320-325, 2000.

5. El-Serag HB, Kramer JR, Chen GJ, Duan Z, Richardson PA and Davila JA: Effectiveness of AFP and ultrasound tests on hepatocellular carcinoma mortality in $\mathrm{HCV}$-infected patients in the USA. Gut 60: 992-997, 2011.

6. Benowitz S: Liver cancer biomarkers struggling to succeed. J Natl Cancer Inst 99: 590-591, 2007.

7. Sherman M: Current status of $\alpha$-fetoprotein testing. Gastroenterol Hepatol (NY) 7: 113-114, 2011.

8. Bruix J and Sherman M; Practice Guidelines Committee, American Association for the Study of Liver Diseases: Management of hepatocellular carcinoma. Hepatology 42: 1208-1236, 2005.

9. Nakamoto Y, Guidotti LG, Kuhlen CV, Fowler P and Chisari FV: Immune pathogenesis of hepatocellular carcinoma. J Exp Med 188: 341-350, 1998.

10. Cacalano NA and Johnston JA: Interleukin-2 signaling and inherited immunodeficiency. Am J Hum Genet 65: 287-293, 1999.

11. Cabrera R, Ararat M, Cao M, et al: Hepatocellular carcinoma immunopathogenesis: clinical evidence for global T cell defects and an immunomodulatory role for soluble CD25 (sCD25). Dig Dis Sci 55: 484-495, 2010.

12. Hoechst B, Ormandy LA, Ballmaier M, et al: A new population of myeloid-derived suppressor cells in hepatocellular carcinoma patients induces CD4(+)CD25(+)Foxp3(+) T cells. Gastroenterology 135: 234-243, 2008. 
13. Arun B, Curti BD, Longo DL, et al: Elevations in serum soluble interleukin-2 receptor levels predict relapse in patients with hairy cell leukemia. Cancer J Sci Am 6: 21-24, 2000.

14. Manshouri T, Do KA, Wang X, et al: Circulating CD20 is detectable in the plasma of patients with chronic lymphocytic leukemia and is of prognostic significance. Blood 101: 2507-2513, 2003.

15. Knodell RG, Ishak KG, Black WC, et al: Formulation and application of a numerical scoring system for assessing histological activity in asymptomatic chronic active hepatitis. Hepatology 1 : 431-435, 1981.

16. Llovet JM, Brú C and Bruix J: Prognosis of hepatocellular carcinoma: the BCLC staging classification. Semin Liver Dis 19: 329-338, 1999.

17. Trevisani F, D'Intino PE, Morselli-Labate AM, et al: Serum alpha-fetoprotein for diagnosis of hepatocellular carcinoma in patients with chronic liver disease: influence of HBsAg and anti-HCV status. J Hepatol 34: 570-575, 2001.

18. Sassa T, Kumada T, Nakano S and Uematsu T: Clinical utility of simultaneous measurement of serum high-sensitivity des-gamma-carboxy prothrombin and Lens culinaris agglutinin A-reactive alpha-fetoprotein in patients with small hepatocellular carcinoma. Eur J Gastroenterol Hepatol 11: 1387-1392, 1999.

19. Shimauchi Y, Tanaka M, Kuromatsu R, et al: A simultaneous monitoring of Lens culinaris agglutinin A-reactive alphafetoprotein and des-gamma-carboxy prothrombin as an early diagnosis of hepatocellular carcinoma in the follow-up of cirrhotic patients. Oncol Rep 7: 249-256, 2000

20. Marrero JA, Su GL, Wei W, et al: Des-gamma carboxyprothrombin can differentiate hepatocellular carcinoma from nonmalignant chronic liver disease in American patients. Hepatology 37: 1114-1121, 2003.

21. Marrero JA, Feng Z, Wang Y, et al: Alpha-fetoprotein, des-gamma carboxy prothrombin, and lectin-bound alpha-fetoprotein in early hepatocellular carcinoma. Gastroenterology 137: 110-118, 2009.

22. Carr BI, Kanke F, Wise M and Satomura S: Clinical evaluation of lens culinaris agglutinin-reactive alpha-fetoprotein and des-gamma-carboxy prothrombin in histologically proven hepatocellular carcinoma in the United States. Dig Dis Sci 52: $776-782,2007$
23. Lok AS, Sterling RK, Everhart JE, et al; HALT-C Trial Group: Des-gamma-carboxy prothrombin and alpha-fetoprotein as biomarkers for the early detection of hepatocellular carcinoma. Gastroenterology 138: 493-502, 2010.

24. Nakamura S, Nouso K, Sakaguchi K, et al: Sensitivity and specificity of des-gamma-carboxy prothrombin for diagnosis of patients with hepatocellular carcinomas varies according to tumor size. Am J Gastroenterol 101: 2038-2043, 2006.

25. Sterling RK, Wright EC, Morgan TR, et al: Frequency of elevated hepatocellular carcinoma (HCC) biomarkers in patients with advanced hepatitis C. Am J Gastroenterol 107: 64-74, 2012.

26. Di Bisceglie AM, Sterling RK, Chung RT, et al; HALT-C Tria Group: Serum alpha-fetoprotein levels in patients with advanced hepatitis C: results from the HALT-C trial. J Hepatol 43: 434-441, 2005.

27. Yoon SK, Lim NK and Ha S: The human cervical cancer oncogene protein is a biomarker for human hepatocellular carcinoma. Cancer Res 64: 5434-5441, 2004.

28. el-Houseini ME, Mohammed MS, Elshemey WM, Hussein TD, Desouky OS and Elsayed AA: Enhanced detection of hepatocellular carcinoma. Cancer Control 12: 248-253, 2005.

29. Yuen MF and Lai CL: Screening for hepatocellular carcinoma: survival benefit and cost-effectiveness. Ann Oncol 14: 1463-1467, 2003.

30. Davila JA, Henderson L, Kramer JR, et al: Utilization of surveillance for hepatocellular carcinoma among hepatitis $C$ virus-infected veterans in the United States. Ann Intern Med 154: 85-93, 2011

31. Daniele B, Bencivenga A, Megna AS and Tinessa V: Alphafetoprotein and ultrasonography screening for hepatocellular carcinoma. Gastroenterology 127 (Suppl 1): S108-S112, 2004.

32. Pepe MS, Etzioni R, Feng Z, et al: Phases of biomarker development for early detection of cancer. J Natl Cancer Inst 93: 1054-1061, 2001 